\title{
An anisotropic elastic-viscoplastic damage model for bone tissue
}

\author{
J. J. Schwiedrzik · P. K. Zysset
}

Received: 29 November 2011 / Accepted: 21 March 2012 / Published online: 18 April 2012

(C) Springer-Verlag 2012

\begin{abstract}
A new anisotropic elastic-viscoplastic damage constitutive model for bone is proposed using an eccentric elliptical yield criterion and nonlinear isotropic hardening. A micromechanics-based multiscale homogenization scheme proposed by Reisinger et al. is used to obtain the effective elastic properties of lamellar bone. The dissipative process in bone is modeled as viscoplastic deformation coupled to damage. The model is based on an orthotropic ecuntric elliptical criterion in stress space. In order to simplify material identification, an eccentric elliptical isotropic yield surface was defined in strain space, which is transformed to a stressbased criterion by means of the damaged compliance tensor. Viscoplasticity is implemented by means of the continuous Perzyna formulation. Damage is modeled by a scalar function of the accumulated plastic strain $D(\kappa)$, reducing all elements of the stiffness matrix. A polynomial flow rule is proposed in order to capture the rate-dependent post-yield behavior of lamellar bone. A numerical algorithm to perform the back projection on the rate-dependent yield surface has been developed and implemented in the commercial finite element solver Abaqus/Standard as a user subroutine UMAT. A consistent tangent operator has been derived and implemented in order to ensure quadratic convergence. Correct implementation of the algorithm, convergence, and accuracy of the tangent operator was tested by means of strain- and stress-based single element tests. A finite element simulation of nano-
\end{abstract}

\footnotetext{
J. J. Schwiedrzik

Institute of Lightweight Design and Structural Biomechanics, Vienna University of Technology, Gusshausstr. 27-29, 1040,

Vienna, Austria

J. J. Schwiedrzik (凶) • P. K. Zysset

Institute of Surgical Technology and Biomechanics, University of Bern, Stauffacherstr. 78, 3014, Bern, Switzerland

e-mail: jakob.schwiedrzik@istb.unibe.ch
}

indentation in lamellar bone was finally performed in order to show the abilities of the newly developed constitutive model.

Keywords Bone - Constitutive model · Viscoplasticity · Damage

\section{Introduction}

Bone is a biomaterial exhibiting complex mechanical behavior, especially in the post-yield regime. In order to be able to make quantitative predictions of bone stiffness and failure, realistic constitutive models of its mechanical behavior are needed. Due to the hierarchical nature of bone, the apparent mechanical properties at different length scales vary. Micromechanical approaches have been applied in the past to predict elastic and strength properties of bone on several length scales. Many of the nonlinear constitutive models proposed so far have concentrated on bone at the macroscopic organ level (Zysset 1994; Fondrk et al. 1999; Keyak and Rossi 2000; Natali et al. 2008; Garcia et al. 2009; Charlebois et al. 2010). Recently, several models have been proposed that describe the behavior of bone during nanoindentation (Tai et al. 2006; Zhang et al. 2008, 2010; Carnelli et al. 2010; Lucchini et al. 2011). Most of these models have been restricted to rateindependent post-yield behavior. It has been shown, though, that bone exhibits a strong strain-rate dependency after yielding (Gupta et al. 2007; Gupta and Zioupos 2008), a phenomenon also seen during nanoindentation experiments at the ultrastructural level where creep behavior may be observed during holding periods (Zysset et al. 1999; Bushby et al. 2004; Wolfram et al. 2010). In this work, a constitutive model for bone has been developed that has the potential to be used on different length scales ranging from the 
ultrastructural to the macroscopic level. A micromechanical approach to assess the elastic properties was combined with a phenomenological constitutive law describing the viscoplastic and damage post-yield behavior of bone. It features anisotropic elasticity, an eccentric elliptical yield surface, viscoplasticity, and damage, i.e., progressive degradation of the stiffness tensor. The purpose of this model is to be able to predict experimental force-displacement curves on several length scales from the ultrastructural to the macroscopical level by using finite element simulation and appropriate material properties. The mathematical formulation of the model is performed within the framework of thermodynamics of irreversible processes. The proposed model uses the internal state variable approach common in continuum mechanics and allows a straightforward interpretation of the constitutive behavior of cortical bone in terms of plastic deformation and damage. It does not account for the damage and inelastic deformation mechanisms in the molecular regime. Instead, several assumptions are made on the shape of the yield surface and the validity of the theory of plasticity and continuum damage mechanics. These assumptions will be listed and justified during the course of this article.

Bone is a hierarchical material with three main constituents: collagen, mineral, and water. Collagen molecules self-assemble into fibrils, which are periodically reinforced by mineral platelets (Weiner and Wagner 1998; Fratzl and Weinkamer 2007), the empty pore space is filled with water. This basic unit forms fibril arrays, bundles of parallel mineralized fibrils embedded in a extra-fibrillar mineral matrix with a foam-like structure (Hellmich and Ulm 2002; Reisinger et al. 2010). In lamellar bone, parallel fibril arrays form lamellae in a rotated plywood-like manner (Weiner et al. 1997, 1999). Multiple bone lamellae arranged around a blood vessel make up an osteon, which features microporosity of up to $10 \%$, mainly of the lacunar-canalicular network (Sugawara et al. 2005). Cortical bone consists mainly of parallel arrays of osteons going in the axial direction of the bone with blood vessels making up for a macroporosity of about $6 \%$ (Fratzl and Weinkamer 2007). For a more thorough description, see e.g., Fratzl and Weinkamer (2007).

As shown in the previous paragraph, there is a considerable amount of porosity present on every hierarchical level of bone from the nano- to the macro-scale. Tai et al. (2006) showed evidence that bone behaves as a cohesive-frictional material due to its nanogranular structure. They proposed that the increased yield properties reported in the literature Yeni et al. (2004) in compression compared to tension may be explained by nanogranular friction between mineral particles and cohesion that originates from within the organic phase itself. Finite element simulations using plasticity models featuring a Drucker-Prager-type yield surface were able to capture some of the characteristics of nanoindentation experiments on bone (Tai et al. 2006; Carnelli et al. 2010). Micromechanical considerations by Maghous et al. (2009) on the strength of porous geomaterials showed that the yield surface of cohesive-frictional materials featuring porosity takes an eccentric elliptical shape. This is also consistent with findings on the macroscopic level, where Cowin proposed a Tsai-Wu yield surface for cortical bone (Cowin 1979). Due to the considerable amount of porosity present on every hierarchical level in bone, we chose to base the model on an eccentric elliptical yield surface in order to make it compatible with multiple length scales from the ultrastructural to the macroscopic level. This is consistent with the notion of bone consisting of a porous mineral nanogranular matrix with organic glue reinforced by collagen fibers.

When loaded past the yield point, bone shows two mechanisms of energy dissipation: inelastic deformation and damage, that is, reduction in the elastic properties. The formation of plastic and damage behavior is well documented on the macroscopic scale (Garcia et al. 2009). Furthermore, it has been shown that bone exhibits a strong strain-rate dependency after yielding on the macro-scale (Gupta et al. 2007; Gupta and Zioupos 2008) that may be captured by a viscoplastic approach. However, there is evidence that these mechanisms exist from the nanoscale upwards (Gupta et al. 2005, 2006; Hansma et al. 2005). Gupta et al. proposed a model where inelastic deformation at the fibril level takes place by viscous flow of the interfibrillar matrix past the fibrils transmitting shear flow stresses, once a certain strain level is reached. This process may be described by viscoplasticity taking into account the time and rate dependency of the postyield process and a strain-based yield criterion. One possible approach is the continuous Perzyna formulation, first proposed by Ponthot (1995) allowing for a smooth transition from rate-independent elastoplasticity to viscoplasticity. Gupta et al. (2006) also interpreted their findings of negative fibril strains after macroscopical inelastic strains in tension as a sign for additional internal decohesion taking place between the mineral and the collagen molecules, which may be interpreted as a formation of damage from a continuum mechanics point of view. Another experimental finding supporting the notion of damage mechanisms present at the ultrastructural level is the decrease of indentation modulus with increasing indentation depth reported in instrumented nanoindentation experiments performed on bone (Hengsberger et al. 2002; Zhang et al. 2008; Voyiadjis and Peters 2010) and the presence of transverse microcracks around the indentation site reported by Hengsberger et al. (2002). Finite element simulations of nanoindentation experiments using coupled plasticity and damage models have shown that the presence of damage may explain some of the experimental findings for mineralized tissues like size effects (Zhang et al. 2010; Lucchini et al. 2011). Therefore, both dissipative mechanisms were included in the model by means of a plastic strain 
tensor and a scalar damage variable reducing all elements of the stiffness tensor as a function of accumulated plastic strain.

The model is based on an orthotropic eccentric elliptical criterion in stress spaces. In order to simplify material identification, a strain-based formulation that is isotropic in strain space was formulated as an approximation of the yield surface of cortical bone. The advantage of this approach is the ability of the model to deal with general anisotropy and the low number of material constants needed. Experimental findings of Gupta et al. support the hypothesis that the yield properties of bone can be described by a surface in strain space on the ultrastructural as well as the macroscopic level (Gupta et al. 2006). Due to the current lack of reliable experimental data on strength properties of lamellar bone on several length scales that would allow us to refine the model, an isotropic eccentric criterion in strain space was postulated that is transformed to stress space using the damaged compliance tensor for use in the stress integration algorithm.

\section{Constitutive model}

The rheological model is a damageable elastic spring in series with a plastic pad and a dashpot element in parallel. In the purely elastic regime, the model behaves independently of the strain rate. The plastic strains are accumulating viscously using a Perzyna-type viscoplasticity formulation. The model is based on the internal variables $\boldsymbol{E}^{p}$ and $\kappa$. The state variable $\boldsymbol{E}^{p}$ is a second-order tensor including the inelastic strains, $\kappa$ is the accumulated plastic strain. Damage accumulation is assumed to be coupled to the plasticity using a damage function $D(\kappa)$ reducing all elements of the stiffness tensor. $D$ is limited between 0 (no damage) and 1 (failure). Figure 1 shows the rheological model for the implemented constitutive law.

\subsection{Free energy potential and dissipation}

In the notation used in the following chapters, scalars are written as $X$, second-order tensors as $\boldsymbol{X}$, and fourth-order tensors as $\mathbb{X}$, ":" denotes the double contraction operation. In case of compositions of two fourth-order tensors $\left(\mathbb{X}_{i j k l}=\right.$

\section{(Damaged) Elastic Spring Hardening/Softening Element}

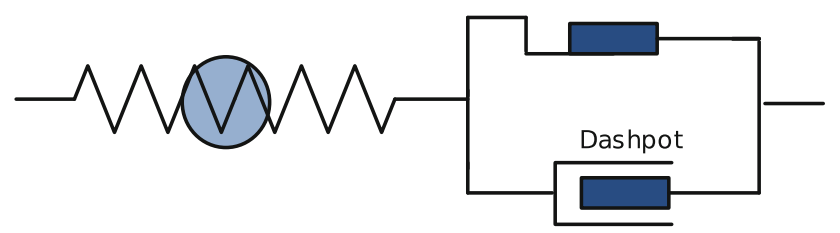

Fig. 1 Rheological model of an elasto-viscoplastic solid with damage
$\left.\mathbb{Y}_{i j m n} \mathbb{Z}_{m n k l}\right)$ and of the transformations of a second-order tensor with a fourth-order tensor $\left(\boldsymbol{X}_{i j}=\mathbb{Y}_{i j k l} \boldsymbol{Z}_{k l}\right)$, the ":" sign is not written. The operator $\otimes$ denotes the dyadic product $\mathbb{X}_{i j k l}=\boldsymbol{Y}_{i j} \boldsymbol{Z}_{k l}, \underline{\bar{\otimes}}$ the symmetric product $\mathbb{X}_{i j k l}=$ $\frac{1}{2}\left(\boldsymbol{Y}_{i k} \boldsymbol{Z}_{j l}+\boldsymbol{Y}_{i l} \boldsymbol{Z}_{j k}\right)$.

The finite total strain tensor is split additively into an elastic and a plastic part using the Green-Naghdi decomposition (Green and Naghdi 1965):

$\boldsymbol{E}=\boldsymbol{E}^{e}+\boldsymbol{E}^{p}$

The cumulated plastic strain $\kappa$ is defined as:

$\kappa=\int_{0}^{t}\left\|\dot{\boldsymbol{E}}^{p}\right\| \mathrm{d} \tau$

In this model, damage is modeled as a scalar $D$ reducing all components of the stiffness tensor. Damage is assumed to be dependent on the history of permanent deformation and therefore defined as a function of the accumulated plastic strain $\kappa$ (Zysset 1994; Charlebois et al. 2010):

$D(\kappa)=1-e^{-k_{p} \kappa}$

The constant $k_{p}$ was set to 10.5 following the findings of Zysset (1994). The free energy potential for this material model was defined as:

$\Psi\left(\boldsymbol{E}, \boldsymbol{E}^{p}, \kappa\right)=\frac{1}{2}(1-D(\kappa))\left(\boldsymbol{E}-\boldsymbol{E}^{p}\right): \mathbb{S}\left(\boldsymbol{E}-\boldsymbol{E}^{p}\right)$

with the stiffness tensor $\mathbb{S}$, the total strains $\boldsymbol{E}$ and the plastic strains $\boldsymbol{E}^{p}$. The corresponding state laws become:

$\boldsymbol{S}_{\Psi}=\nabla_{\boldsymbol{E}} \Psi=(1-D(\kappa)) \mathbb{S}\left(\boldsymbol{E}-\boldsymbol{E}^{p}\right)$

and

$\boldsymbol{S}_{\Psi}^{p}=-\nabla_{\boldsymbol{E}^{p}} \Psi=(1-D(\kappa)) \mathbb{S}\left(\boldsymbol{E}-\boldsymbol{E}^{p}\right)$

and

$$
\begin{aligned}
W_{\Psi}^{\kappa} & =-\nabla_{\kappa} \Psi \\
& = \begin{cases}\frac{1}{2} D^{\prime}(\kappa)\left(\boldsymbol{E}-\boldsymbol{E}^{p}\right): \mathbb{S}\left(\boldsymbol{E}-\boldsymbol{E}^{p}\right) & \text { if } \kappa \in] 0, \infty[ \\
0 & \text { if } \kappa=0 .\end{cases}
\end{aligned}
$$

where $S$ is the stress tensor. The conjugate variables are $S$ and $\boldsymbol{E}, \boldsymbol{S}^{p}$ and $\boldsymbol{E}^{p}$ as well as $W^{\kappa}$ and $\kappa$. For the model to be thermodynamically admissible, the dissipation $\Phi$ needs to be positive at all times. The dissipation can be expressed as the difference between the stress power density and the rate of the free energy density:

$\Phi=S: \dot{\boldsymbol{E}}-\dot{\Psi}$

The dissipation becomes therefore:

$\Phi=\boldsymbol{S}: \dot{\boldsymbol{E}}-\boldsymbol{S}: \dot{\boldsymbol{E}}+\boldsymbol{S}^{p}: \dot{\boldsymbol{E}}^{p}+W^{\kappa} \dot{\kappa}$ 
The first two terms cancel each other out and the dissipation becomes

$$
\begin{aligned}
\Phi= & (1-D(\kappa)) \mathbb{S}\left(\boldsymbol{E}-\boldsymbol{E}^{p}\right): \dot{\boldsymbol{E}}^{p} \\
& +\frac{1}{2} D^{\prime}(\kappa)\left(\boldsymbol{E}-\boldsymbol{E}^{p}\right): \mathbb{S}\left(\boldsymbol{E}-\boldsymbol{E}^{p}\right) \dot{\kappa}
\end{aligned}
$$

with a plastic and a damage contribution to the overall dissipation.

\subsection{Viscoplastic formulation}

Similar to the governing equations of the flow theory in rateindependent plasticity, the constitutive relations of an elastic-viscoplastic material of the Perzyna type can be written as (Perzyna 1966; Etse and Carosio 1999):

$\boldsymbol{S}=(1-D(\kappa)) \mathbb{S}\left(\boldsymbol{E}-\boldsymbol{E}^{p}\right)$

$\dot{\boldsymbol{E}}^{p}=\frac{1}{\eta}\langle\psi(Y)\rangle \boldsymbol{M}^{p}$

$\boldsymbol{M}^{p}=\nabla_{S} Y$

with the yield function $Y=Y(\boldsymbol{S}, \kappa)$. The \langle\rangle are the McAuley brackets in their usual meaning $\langle f(x)\rangle=\frac{1}{2}(f(x)+$ $|f(x)|)$. Following the suggestion of Ponthot (1995), a viscoplastic consistency parameter $\dot{\lambda}$ is introduced. This approach is known as the continuous Perzyna formulation.

$\dot{\lambda}=\frac{1}{\eta}\langle\psi(Y)\rangle$

$\psi(Y)$ is a monotonously increasing, invertible function. By substituting the consistency parameter into the viscoplastic flow rules, they take a form well known from rate-independent plasticity:

$\dot{\boldsymbol{E}}^{p}=\dot{\lambda} \boldsymbol{M}^{p}$

For viscoplastic materials of the Perzyna type, the stress state can lie outside of the rate-independent yield surface during viscoplastic flow. In the inelastic regime $(Y \geq 0)$, the overstress function $\psi(Y)$ is invertible and the yield function follows its inverse:

$Y=\psi^{-1}(\dot{\lambda} \eta)$

Therefore, we can define a new condition constraining the viscoplastic flow:

$\bar{Y}=Y-\psi^{-1}(\dot{\lambda} \eta)=0$

According to Etse and Carosio (1999), Carosio et al. (2000), this condition represents a generalization of the rate-independent yield condition $Y=0$ for viscoplastic materials of the Perzyna type. It allows the use of generalized Kuhn-Tucker conditions (Kuhn and Tucker 1951) for viscoplastic flow in the form of

$\bar{Y} \leq 0, \quad \dot{\lambda} \geq 0, \quad \dot{\lambda} \bar{Y}=0$, which assure that the inelastic process satisfies $\bar{Y}=0$ during viscoplastic deformation and that no permanent deformation occurs in the elastic regime. This means that during viscoplastic deformation, the generalized consistency condition (Simo and Ju 1987; Chaboche 2008) holds true:

$\dot{\bar{Y}}=0$

It should be noted that in this approach for $\eta \rightarrow 0$, the rate-dependent yield surface $\bar{Y}$ degenerates to the rateindependent $Y$, transforming the elasto-viscoplastic model to an elastoplastic one.

\subsection{Continuum tangent operator}

The continuum tangent stiffness operator gives the relationship between the stress rate $\dot{S}$ and the strain rate $\dot{\boldsymbol{E}}$ in the continuum rate equation:

$\dot{\boldsymbol{S}}=\mathbb{S}_{C} \dot{\boldsymbol{E}}$

In the elastic case $(\bar{Y}<0)$, the continuum tangent has the form

$\mathbb{S}_{C, e l}=(1-D(\kappa)) \mathbb{S}$

Differentiation of (1), (11), (14), and (17) with respect to time provides the following set of equations to evaluate the tangent in the case $\bar{Y}=0$ :

$$
\begin{aligned}
\dot{\boldsymbol{E}} & =\dot{\boldsymbol{E}}^{e}+\dot{\boldsymbol{E}}^{p} \\
\dot{\boldsymbol{S}} & =-D^{\prime}(\kappa) \mathbb{S}\left(\boldsymbol{E}-\boldsymbol{E}^{p}\right) \dot{\kappa}+(1-D(\kappa)) \mathbb{S}\left(\dot{\boldsymbol{E}}-\dot{\boldsymbol{E}}^{p}\right) \\
\dot{\bar{Y}} & =\dot{Y}-\dot{\psi}^{-1}=\left(\nabla_{\boldsymbol{S}} Y\right) \dot{\boldsymbol{S}}+\frac{\partial Y}{\partial \kappa} \dot{\kappa}-\frac{\partial \psi^{-1}}{\partial \dot{\lambda}} \ddot{\lambda}=0 \\
\ddot{\lambda} & =\frac{1}{\eta} \psi^{\prime}(Y) \dot{Y}=\frac{1}{\eta} \psi^{\prime}(Y)\left(\left(\nabla_{S} Y\right) \dot{\boldsymbol{S}}+\frac{\partial Y}{\partial \kappa} \dot{\kappa}\right)
\end{aligned}
$$

For the exact form of the gradients and derivatives, see the "Appendix. By combining (22) and (23) with (11) and substituting $\dot{\lambda}=\frac{\dot{\kappa}}{\left\|\nabla_{S} Y\right\|}$, we get:

$\dot{\boldsymbol{E}}=\frac{\mathbb{E} \dot{\boldsymbol{S}}}{1-D(\kappa)}+\frac{\mathbb{E} \boldsymbol{S}}{(1-D(\kappa))^{2}} D^{\prime}(\kappa) \dot{\kappa}+\dot{\kappa} \boldsymbol{N}^{p}$

with the compliance tensor $\mathbb{E}=\mathbb{S}^{-1}$ and

$N^{p}=\frac{\nabla_{S} Y}{\left\|\nabla_{S} Y\right\|}$

Combining (24) and (25) provides the following equation:

$\left(\nabla_{S} Y \dot{S}+\frac{\partial Y}{\partial \kappa} \dot{\kappa}\right)\left(1-\frac{1}{\eta} \frac{\partial \psi^{-1}}{\partial \dot{\lambda}} \frac{\partial \psi}{\partial Y}\right)=0$

For $\eta \rightarrow 0$, the first expression has to vanish due to the consistency condition $\dot{Y}=0$ for rate-independent materials. This provides a relationship between $\dot{\kappa}$ and $\dot{S}$. Substituting this into (26) and accounting for (11) leads to: 


$$
\begin{aligned}
\dot{\boldsymbol{E}}= & \left(\frac{\mathbb{E}}{1-D(\kappa)}-\left(\boldsymbol{N}^{p} \otimes \boldsymbol{N}^{p}\right.\right. \\
& \left.\left.+\frac{D^{\prime}(\kappa)}{1-D(\kappa)}\left(\boldsymbol{E}-\boldsymbol{E}^{p}\right) \otimes \boldsymbol{N}^{p}\right) \frac{\left\|\nabla_{\boldsymbol{S}} Y\right\|}{\frac{\partial Y}{\partial \kappa}}\right) \dot{\boldsymbol{S}}
\end{aligned}
$$

The continuum tangent operator for the rate-independent material is therefore given by:

$$
\begin{aligned}
\mathbb{S}_{C}= & \left(\frac{\mathbb{E}}{1-D(\kappa)}-\left(\boldsymbol{N}^{p} \otimes \boldsymbol{N}^{p}\right.\right. \\
& \left.\left.+\frac{D^{\prime}(\kappa)}{1-D(\kappa)}\left(\boldsymbol{E}-\boldsymbol{E}^{p}\right) \otimes \boldsymbol{N}^{p}\right) \frac{\left\|\nabla_{S} Y\right\|}{\frac{\partial Y}{\partial \kappa}}\right)^{-1}
\end{aligned}
$$

It features a damaged elastic stiffness, a rank one correction term connected to the associated plasticity and a second correction term accounting for the damage accumulation. The presence of damage makes the problem non-associated, the tangent operator loses major symmetry. By applying the Sherman-Morrison formula, a straightforward expression for the continuum tangent stiffness of the rate-independent case may be obtained:

$$
\begin{aligned}
& \mathbb{S}_{C}=(1-D(\kappa)) \mathbb{S} \\
& -\frac{(1-D(\kappa))^{2} \mathbb{S}\left(\boldsymbol{N}^{p}+\frac{D^{\prime}(\kappa)}{1-D(\kappa)}\left(\boldsymbol{E}-\boldsymbol{E}^{p}\right)\right) \otimes \boldsymbol{N}^{p} \mathbb{S}}{(1-D(\kappa)) \boldsymbol{N}^{p} \mathbb{S}\left(\boldsymbol{N}^{p}+\frac{D^{\prime}(\kappa)}{1-D(\kappa)}\left(\boldsymbol{E}-\boldsymbol{E}^{p}\right)\right)-\frac{1}{\left\|\nabla_{S} Y\right\|} \frac{\partial Y}{\partial \kappa}}
\end{aligned}
$$

If damage and isotropic hardening are turned off, the tangent degenerates to:

$\mathbb{S}_{C, e p}=\mathbb{S}-\frac{\mathbb{S} N^{p} \otimes N^{p} \mathbb{S}}{N^{p} \mathbb{S} N^{p}}$

which is equivalent to the classical tangent elastoplastic tensor (Zinkiewicz et al. 1969; Rakatomanana et al. 1991). For the viscoplastic material $(\eta \neq 0)$, the first expression of (27) does not vanish and therefore the second one has to be equal to 0 . This leads to a differential equation that can be solved for special cases as demonstrated by Carosio et al. (2000). A general solution for this problem is beyond the scope of this article. However, an algorithmic tangent will be presented for the rate-dependent case later in this article.

\subsection{Elastic stiffness}

The proposed constitutive model can handle elastic tensors with material symmetries ranging from isotropy to general anisotropy. In this work, we propose to base the elastic properties on a multiscale homogenization scheme for lamellar bone proposed by Reisinger et al. (2010, 2011).The scheme starts by modeling the mineralized fibrils as a collagen matrix reinforced by ellipsoid mineral inclusions and the extrafibrillar matrix as a mineral matrix with spherical pores. In a second homogenization step, the fibril array is modeled as an extra-fibrillar matrix reinforced by mineralized collagen fibrils. Both homogenization steps are performed using a Mori-Tanaka scheme (Nemat-Nassar and Mori 1993). The resulting stiffness tensor for a single fibril array is transversely isotropic. In a third step, a laminate unit cell describing a single bone lamella is built and periodic boundary conditions are applied. The stiffness tensor of the unit cell is determined by applying 6 independent load cases. The resulting stiffness operator can have material symmetries ranging from isotropy to general anisotropy depending on the sublamellar arrangement of fibril arrays. For further details, see Reisinger et al. (2010, 2011). Recently, an attempt has been made to validate the fibril array model experimentally on mineralized turkey leg tendon by Spiesz et al. (2011), Spiesz (2011). Comparison to experimental results on two length scales obtained through nanoindentation and comparison of macroscopical tension tests to $\mu \mathrm{FE}$ simulations showed that the model is able to predict the anisotropic stiffness of uniaxially aligned fibril arrays if the needed parameters are obtained locally at a sufficient accuracy.

\subsection{Yield criterion}

The model is based on an eccentric elliptical criterion in stress space featuring isotropic hardening and a back stress $\boldsymbol{A}$. It takes the form of:

$Y(\boldsymbol{S}, \kappa):=\sqrt{(\boldsymbol{S}-r(\kappa) \boldsymbol{A}): \mathbb{A}(\boldsymbol{S}-r(\kappa) \boldsymbol{A})}-r(\kappa)$

The fourth-order tensor $\mathbb{A}$ and the back stress $\boldsymbol{A}$ can be determined from an equivalent orthotropic Tsai-Wu criterion using a transformation introduced by Shih and Lee (1978):

$$
\begin{aligned}
\mathbb{A} & =\frac{\mathbb{F}}{1+\boldsymbol{A} \mathbb{F} \boldsymbol{A}} \\
\boldsymbol{A} & =-\frac{1}{2} \mathbb{F}^{-1} \boldsymbol{F}
\end{aligned}
$$

The general forms of the tensors $\mathbb{F}$ and $\boldsymbol{F}$ defining the Tsai-Wu criterion are:

$$
\begin{aligned}
\mathbb{F}= & \sum_{i=1}^{3} \frac{1}{\sigma_{i}^{+} \sigma_{i}^{-}} \boldsymbol{M}_{i} \otimes \boldsymbol{M}_{i}-\sum_{i, j=1 ; i \neq j}^{3} \frac{\zeta_{i j}}{\sigma_{i}^{+} \sigma_{i}^{-}} \boldsymbol{M}_{i} \otimes \boldsymbol{M}_{j} \\
& +\sum_{i, j=1 ; i \neq j}^{3} \frac{1}{2 \tau_{i j}^{2}} \boldsymbol{M}_{i} \underline{\bar{\otimes}} \boldsymbol{M}_{j}
\end{aligned}
$$

and

$$
\boldsymbol{F}=\sum_{i=1}^{3}\left(\frac{1}{\sigma_{i}^{+}}-\frac{1}{\sigma_{i}^{-}}\right) \boldsymbol{M}_{i}
$$

with

$$
\boldsymbol{M}_{i}=\boldsymbol{m}_{i} \otimes \boldsymbol{m}_{i}
$$


The $\boldsymbol{m}_{i}$ are the vectors describing the material orientation. Twelve orthotropic constants are needed to define the criterion: $\sigma_{1}^{+}, \sigma_{1}^{-}, \sigma_{2}^{+}, \sigma_{2}^{-}, \sigma_{3}^{+}, \sigma_{1}^{-}, \zeta_{12}, \zeta_{23}, \zeta_{31}, \tau_{12}, \tau_{23}$, and $\tau_{31}$. It shows isotropic hardening with respect to the origin of stress space rather than the midpoint of the ellipse. This is done in order to ensure that the tension/compression yield stress ratio is not altered by the isotropic hardening. In this formulation, the hardening function is hypothesized to be an explicit function of the accumulated plastic strain.

$r(\kappa):=1+\left({ }^{y} r-1\right) g(\kappa)$

with $g(\kappa)$ being an arbitrary (nonlinear) function of $\kappa$. The scalar ${ }^{y} r$ describes the ratio of the yield and ultimate properties. In the case of exponential hardening, $g(\kappa)$ takes the form of:

$g(\kappa):=1-e^{-k_{s} \kappa}$

In order to simplify material identification, it was hypothesized that an eccentric isotropic elliptical yield surface in strain space can describe the yield limit of cortical behavior accurately. It features an offset between the isotropic tensile and compressive yield strains. The criterion is given by:

$$
\begin{aligned}
Y_{T W}^{\text {Strain }}\left(\boldsymbol{E}^{e}\right):= & (1-D(\kappa)) \boldsymbol{P}: \boldsymbol{E}^{e} \\
& +\boldsymbol{E}^{e}:(1-D(\kappa))^{2} \mathbb{P} \boldsymbol{E}^{e}-1
\end{aligned}
$$

The general form of the fourth-order tensor $\mathbb{P}$ is:

$$
\begin{aligned}
\mathbb{P}= & \sum_{i=1}^{3} \frac{1}{\epsilon_{0}^{+} \epsilon_{0}^{-}} \boldsymbol{M}_{i} \otimes \boldsymbol{M}_{i}+\sum_{i, j=1 ; i \neq j}^{3} \frac{\xi_{0}}{\epsilon_{0}^{+} \epsilon_{0}^{-}} \boldsymbol{M}_{i} \otimes \boldsymbol{M}_{j} \\
& +\sum_{i, j=1 ; i \neq j}^{3} \frac{2}{\gamma_{0}^{2}} \boldsymbol{M}_{i} \underline{\bar{\otimes}} \boldsymbol{M}_{j}
\end{aligned}
$$

The general form of the second-order tensor $\boldsymbol{P}$ is:

$\boldsymbol{P}=\sum_{i=1}^{3}\left(\frac{1}{\epsilon_{0}^{+}}-\frac{1}{\epsilon_{0}^{-}}\right) \boldsymbol{M}_{i}$

The constants defining the elliptical strain-based criterion for cortical bone were estimated from the experimental findings of Garcia (2006) (Table 1).

The isotropy condition $\mathbb{P}_{1122}=\mathbb{P}_{1111}-\mathbb{P}_{1212}($ Cowin and Mehrabadi 1995) yields:

$\gamma_{0}=\sqrt{\frac{2 \epsilon_{0}^{+} \epsilon_{0}^{-}}{1-\xi_{0}}}$

Table 1 Yield constants for lamellar bone

\begin{tabular}{lll}
\hline$\epsilon_{0}^{+}$ & $\epsilon_{0}^{-}$ & $\xi_{0}$ \\
\hline 0.006 & 0.009 & 0.25 \\
\hline
\end{tabular}

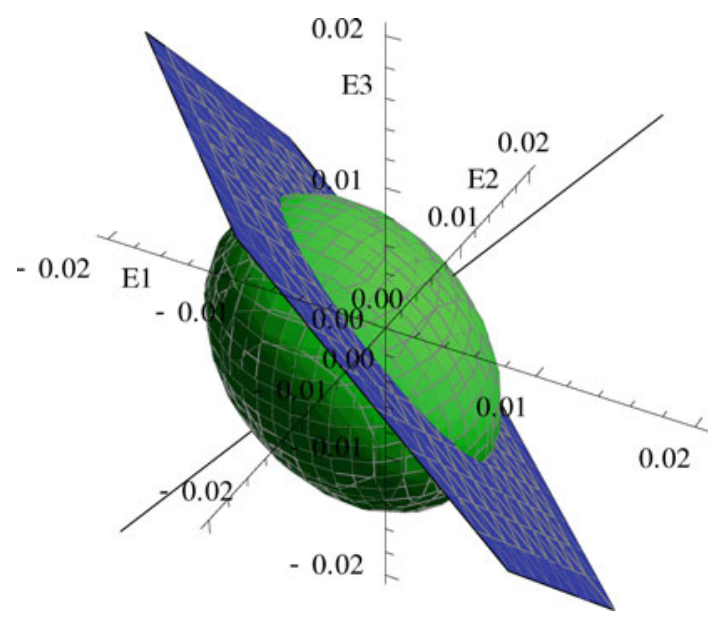

Fig. 2 Elliptical yield criterion for lamellar bone in strain space

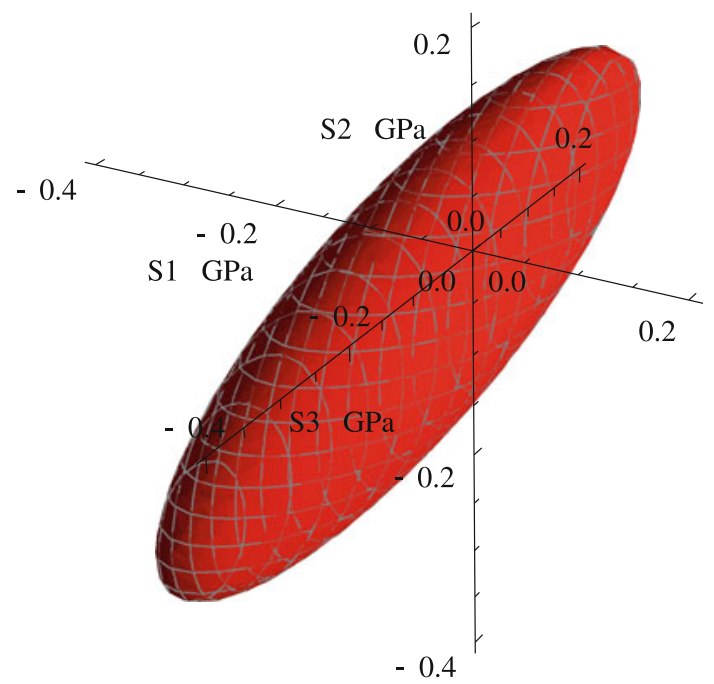

Fig. 3 Elliptical yield criterion for lamellar bone in stress space

The resulting yield surface for lamellar bone in strain space is shown in Fig. 2. The yield criterion is then transformed to stress space using the damaged compliance tensor:

$Y_{T W}^{\text {Stress }}(\boldsymbol{S})=\boldsymbol{P}: \mathbb{E} \boldsymbol{S}+\boldsymbol{S}: \mathbb{E} \mathbb{P} \mathbb{E}-1$

Clearly, the shape of the criterion in stress space is highly dependent on the elasticity tensor. Figure 3 shows an example of a yield surface in stress space for lamellar bone.

The formulation of the elliptical criterion in stress space is similar to the Tsai-Wu criterion, which is originally a failure criterion for composite materials. It is therefore transformed to the Hill criterion defined in (33) by an adaption of the methodology proposed by Shih and Lee (1978):

$\begin{aligned} \mathbb{A} & =\frac{\mathbb{E} \mathbb{P}}{1+\boldsymbol{A}: \mathbb{E} \mathbb{E} \boldsymbol{A}} \\ \boldsymbol{A} & =-\frac{1}{2}(\mathbb{E P E})^{-1} \boldsymbol{P} \mathbb{E}\end{aligned}$ 


\subsection{Numerical algorithm}

In the following chapter, the local iteration number will be called $i$ and should not be mistaken with the increment number $n$. For the sake of simplicity, all state variables at the end of the increment $X_{n+1}$ will be called $X$ and state variables at the beginning of the increment $X_{n}$ will be called $X_{0}$ from now on. The commercial finite element solver Abaqus uses an updated Lagrangian mapping technique with the Cauchy stress tensor and an approximation of the integral of the rate of deformation $\int \boldsymbol{D} \mathrm{d} t$ as the conjugate strain tensor for simulations involving large deformations and rotations. These stress and strain measures were therefore used in this implementation. In principle, the model is consistent for any conjugate pair of stress and strain measures, though.

First, the elastic trial stress is calculated:

$$
\boldsymbol{S}_{T}=\left(1-D\left(\kappa_{0}\right)\right) \mathbb{S}\left(\boldsymbol{E}-\boldsymbol{E}_{0}^{p}\right)
$$

If the yield criterion evaluated using the elastic trial stress and the old damage state is $Y\left(\boldsymbol{S}_{T} ; \kappa_{0}\right)<0$, the stress increment is purely elastic and no further damage is taking place. Therefore, the state variables are updated as follows:

$\kappa=\kappa_{0}$

$\boldsymbol{E}^{p}=\boldsymbol{E}_{0}^{p}$

$S=S_{T}$

The tangent stiffness operator in the elastic case is given by:

$\mathbb{S}_{C A, e l}=\nabla_{E} \boldsymbol{S}=(1-D(\kappa)) \mathbb{S}$

If the yield criterion $Y\left(\boldsymbol{S}_{T} ; \kappa_{0}\right) \geq 0$, an implicit backprojection on the rate-dependent yield surface $\bar{Y}(\boldsymbol{S}, \kappa ; \dot{\lambda})$ is performed. The following set of nonlinear equations needs to be solved using the Newton-Raphson algorithm:

$\boldsymbol{S}=(1-D(\kappa)) \mathbb{S}\left(\boldsymbol{E}-\boldsymbol{E}^{p}\right)$

$\bar{Y}(\boldsymbol{S}, \kappa, \dot{\lambda})=Y(\boldsymbol{S}, \kappa)-\psi^{-1}(\dot{\lambda} \eta)=0$

$\dot{\boldsymbol{E}}^{p}=\dot{\lambda} \boldsymbol{M}^{p}(\boldsymbol{S}, \kappa), \quad \boldsymbol{M}^{p}(\boldsymbol{S}, \kappa)=\nabla_{\boldsymbol{S}} Y$

$\dot{\kappa}=h(\boldsymbol{S}, \kappa) \dot{\lambda}, \quad h(\boldsymbol{S}, \kappa)=\left\|\boldsymbol{M}^{p}\right\|$

constrained by the generalized Kuhn-Tucker conditions

$\bar{Y} \leq 0, \quad \dot{\lambda} \geq 0, \quad \dot{\lambda} \bar{Y}=0$.

The gradients and derivatives can be found in the "Appendix". The viscoplastic consistency parameter $\dot{\lambda}$ is discretized and approximated by $\dot{\lambda}=\frac{\Delta \lambda}{\Delta t}$. The incremental consistency parameter can be written in terms of the incremental change of cumulated plastic strain:

$\Delta \lambda=\frac{\Delta \kappa}{h\left(S, \kappa_{0}+\Delta \kappa\right)}$
The total strains can be rewritten as:

$\boldsymbol{E}=\boldsymbol{E}_{0}+\Delta \boldsymbol{E}$

The stress is linearized and expressed in incremental form as:

$$
\begin{aligned}
\boldsymbol{S}= & \left(1-D\left(\kappa_{0}\right)\right. \\
& \left.-\left(D(\kappa)-D\left(\kappa_{0}\right)\right)\right) \mathbb{S}\left(\boldsymbol{E}_{0}+\Delta \boldsymbol{E}-\boldsymbol{E}_{0}^{p}-\Delta \boldsymbol{E}^{p}\right) \\
= & \boldsymbol{S}_{T}-(D(\kappa) \\
& \left.-D\left(\kappa_{0}\right)\right) \mathbb{S}\left(\boldsymbol{E}-\boldsymbol{E}_{0}^{p}\right)-(1-D(\kappa)) \mathbb{S} \Delta \kappa \boldsymbol{N}^{p}
\end{aligned}
$$

By bringing all expressions onto one side and multiplying with $\frac{\mathbb{E}}{1-D}$, a tensor function expressing the residual error of elastic strains is introduced:

$$
\begin{aligned}
\boldsymbol{R}(\boldsymbol{S}, \Delta \kappa)= & \frac{\mathbb{E}}{1-D\left(\kappa_{0}+\Delta \kappa\right)}\left(\boldsymbol{S}-\boldsymbol{S}^{T}\right) \\
& +\frac{D\left(\kappa_{0}+\Delta \kappa\right)-D\left(\kappa_{0}\right)}{1-D\left(\kappa_{0}+\Delta \kappa\right)}\left(\boldsymbol{E}-\boldsymbol{E}_{0}^{p}\right)+\Delta \kappa \boldsymbol{N}^{p}
\end{aligned}
$$

The rate-dependent yield function $\bar{Y}$ is approximated by

$\bar{Y}(\boldsymbol{S}, \Delta \kappa)=Y\left(\boldsymbol{S}, \kappa_{0}+\Delta \kappa\right)$

$$
-\Psi^{-1}\left(\frac{\Delta \kappa}{h\left(S, \kappa_{0}+\Delta \kappa\right) \Delta t}\right)=0
$$

The discretized equations for $\bar{Y}, \boldsymbol{R}$ are linearized with respect to their variables $S$ and $\Delta \kappa$. The total strain at the end of the increment $\boldsymbol{E}$ is known a priori. This provides a linearized system of equations for the Newton-Raphson algorithm:

$$
\begin{gathered}
\boldsymbol{R}_{i+1}=\boldsymbol{R}_{i}+\nabla_{S} \boldsymbol{R}_{i}: \delta \boldsymbol{S}+\frac{\partial \boldsymbol{R}_{i}}{\partial \Delta \kappa} \delta \Delta \kappa=\mathbf{0} \\
\bar{Y}_{i+1}=\bar{Y}_{i}+\nabla_{S} \bar{Y}_{i} \delta \boldsymbol{S}+\frac{\partial \bar{Y}_{i}}{\partial \Delta \kappa} \delta \Delta \kappa=0
\end{gathered}
$$

The set of equations is solved by determining

$$
\begin{gathered}
\delta \Delta \kappa=-\frac{\frac{1}{\left\|\bar{\nabla}_{S} \bar{Y}_{i}\right\|} \bar{Y}_{i}+\boldsymbol{N}_{i}^{p} \mathbb{S}_{a} \boldsymbol{R}_{i}}{\boldsymbol{N}_{i}^{p} \mathbb{S}_{a}^{E} \frac{\partial \boldsymbol{R}_{i}}{\partial \Delta \kappa}+\frac{1}{\left\|\nabla_{S} \bar{Y}_{i}\right\|} \frac{\partial \bar{Y}_{i}}{\partial \Delta \kappa}} \\
\delta \boldsymbol{S}=\mathbb{S}_{a}\left(\boldsymbol{R}_{i}+\frac{\partial \boldsymbol{R}_{i}}{\partial \Delta \kappa} \delta \Delta \kappa\right)
\end{gathered}
$$

with the algorithmic stiffness tensor

$\mathbb{S}_{a}=-\left(\nabla_{S} \boldsymbol{R}\right)^{-1}$

in an iterative fashion until the norm of the residual in elastic strains $\boldsymbol{R}$ and the rate-dependent yield function $\bar{Y}$ are smaller than a predefined tolerance. After each iteration, the following update is performed:

$$
\begin{aligned}
\boldsymbol{S}_{i+1} & =\boldsymbol{S}_{i}+\delta \boldsymbol{S} \\
\Delta \kappa_{i+1} & =\Delta \kappa_{i}+\delta \Delta \kappa
\end{aligned}
$$




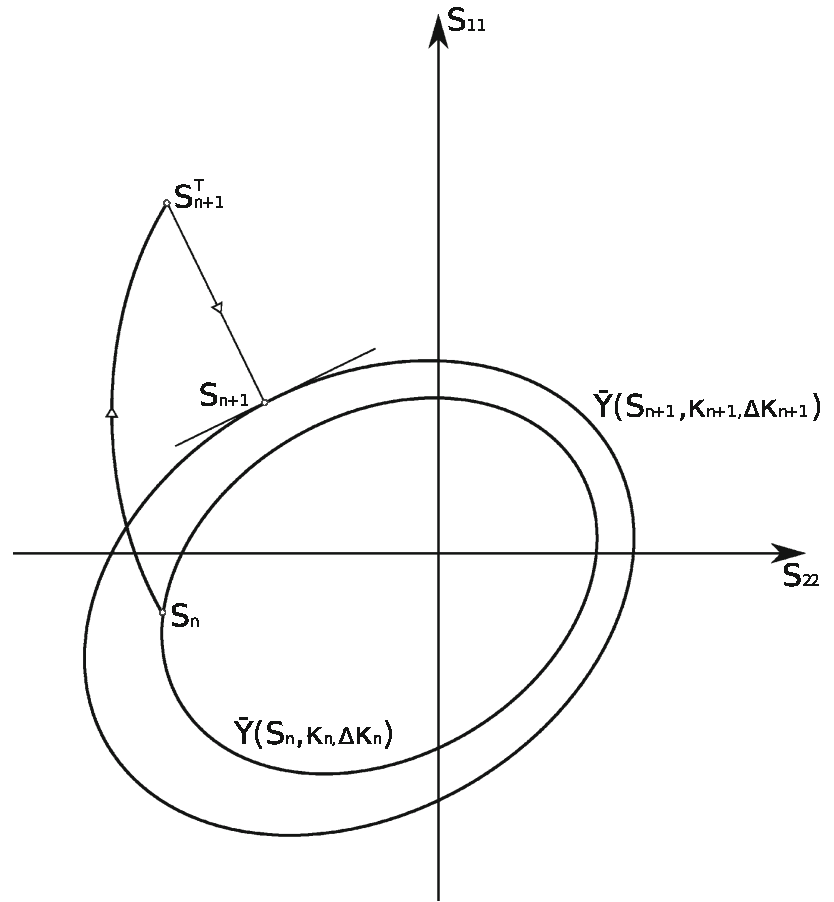

Fig. 4 Schematic sketch of the stress return algorithm

This algorithm corresponds to a projection on the rate-dependent yield criterion $\bar{Y}$. Figure 4 shows a schematic sketch of the implemented stress return algorithm.

After convergence of the algorithm, the state variables at the end of the increment are obtained:

$S=S_{i+1}$

$\kappa=\kappa_{0}+\Delta \kappa_{i+1}$

$\boldsymbol{E}^{p}=\boldsymbol{E}-\frac{\mathbb{E} \boldsymbol{S}}{1-D(\kappa)}$

\subsection{Algorithmic tangent operator}

The algorithmic stiffness tensor $\mathbb{S}_{a}$ relates infinitesimal changes of the strain increment $\delta \Delta \boldsymbol{E}$ to corresponding infinitesimal changes in the stress increment $\delta \Delta \boldsymbol{S}$. In order to find the tangent, a linearization of the stress-strain relationship has to be performed around the current solution. In this case, the consistency condition has to be applied to the algorithmic form of the rate equations. This was already done during the stress integration and does not need to be repeated. After convergence of the Newton scheme, the rate-dependent yield function $\bar{Y}$ is equal to 0 and the residual strain tensor $\boldsymbol{R}$ may be interpreted as an infinitesimal change in strain. Therefore, the tensor relating infinitesimal changes of strain $\delta \boldsymbol{R}=\delta \Delta \boldsymbol{E}$ to infinitesimal changes of the stress increment $\delta \Delta S$ is the sought algorithmic tangent stiffness tensor. In order to find it, the solution for $\left.\delta \Delta \kappa\right|_{\bar{Y}=0}$ has to be substituted into the equation for $\delta S$. The resulting algorithmic tangent stiffness operator is:
$\mathbb{S}_{C A}=\mathbb{S}_{a}-\frac{\mathbb{S}_{a}\left(\frac{\partial \boldsymbol{R}}{\partial \Delta \kappa} \otimes \boldsymbol{N}^{p}\right) \mathbb{S}_{a}}{\boldsymbol{N}^{p} \mathbb{S}_{a} \frac{\partial \boldsymbol{R}}{\partial \Delta \kappa}-\frac{1}{\left\|\nabla_{S} \bar{Y}_{i}\right\|} \frac{\partial \bar{Y}_{i}}{\partial \Delta \kappa}}$

The gradients and derivatives appearing in this expression can be found in the "Appendix". For $\frac{\eta}{\Delta t} \rightarrow 0$, the inverse overstress function $\psi^{-1}(\dot{\lambda} \eta)=0$ and the rate-dependent yield surface $\bar{Y}$ degenerate to the rate-independent $Y$. In this case, the tangent stiffness tensor degenerates to the rate-independent one:

$\mathbb{S}_{C A \mid \frac{\eta}{\Delta t} \rightarrow 0}=\mathbb{S}_{a}-\frac{\mathbb{S}_{a}\left(\frac{\partial \boldsymbol{R}}{\partial \Delta \kappa} \otimes \boldsymbol{N}^{p}\right) \mathbb{S}_{a}}{\boldsymbol{N}^{p}: \mathbb{S}_{a} \frac{\partial \boldsymbol{R}}{\partial \Delta \kappa}-\frac{1}{\left\|\nabla_{S} Y_{i}\right\|} \frac{\partial Y_{i}}{\partial \Delta \kappa}}$

The difference between the algorithmic and the continuum tangent should vanish when the plastic increment $\Delta \kappa \rightarrow 0$. In the case of $\frac{\eta}{\Delta t} \rightarrow 0$, the following relations hold:

$$
\begin{aligned}
& \left.\nabla_{S} \bar{Y}\right|_{\frac{\eta}{\Delta t} \rightarrow 0, \Delta \kappa \rightarrow 0}=\nabla_{S} Y \\
& \left.\frac{\partial \bar{Y}}{\partial \Delta \kappa}\right|_{\frac{\eta}{\Delta t} \rightarrow 0, \Delta \kappa \rightarrow 0}=\frac{\partial Y}{\partial \kappa} \\
& \left.\frac{\partial \boldsymbol{R}}{\partial \Delta \kappa}\right|_{\frac{\eta}{\Delta t} \rightarrow 0, \Delta \kappa \rightarrow 0}=N^{p}+\frac{D^{\prime}(\kappa)}{1-D(\kappa)}\left(\boldsymbol{E}-\boldsymbol{E}^{p}\right)
\end{aligned}
$$

Therefore, as expected, the algorithmic tangent degenerates to the continuum operator for a rate-independent material and infinitesimal plastic strain increments.

$\left.\mathbb{S}_{C A}\right|_{\Delta \kappa \rightarrow 0}=\mathbb{S}_{C}$

For $\frac{\eta}{\Delta t} \rightarrow \infty$, the expression $\frac{1}{\left\|\nabla_{S} \bar{Y}_{i}\right\|} \frac{\partial \bar{Y}_{i}}{\partial \Delta \kappa} \rightarrow \infty$. In this case, the tangent operator degenerates to the elastic one:

$$
\begin{aligned}
\mathbb{S}_{C A \mid \frac{\eta}{\Delta t} \rightarrow \infty} & =-\left(\nabla_{\boldsymbol{S}} \boldsymbol{R}\right)^{-1} \mid \frac{\eta}{\Delta t} \rightarrow \infty \\
& =\left(\frac{\mathbb{E}}{1-D}\right)^{-1}=(1-D) \mathbb{S}
\end{aligned}
$$

The implemented material model therefore shows a smooth transition from rate-independent plasticity to viscoplasticity and ultimately elasticity.

\section{Verification}

\subsection{Single element tests}

Different boundary conditions were imposed in strain- and stress-controlled single element tests using an orthotropic elasticity tensor and an exponential hardening function. Uniaxial tension and compression in all main directions as well as triaxial compression and shear were tested. The strain/stress was increased at a linear rate until a maximum, followed by linear unloading. Stiffness and yield point were checked to verify the correct implementation of the algorithm. The postyield behavior was checked qualitatively. The model showed the expected behavior. The tests converged up to large strains 
for all load cases in strain control. Also, the algorithmic tangent operator was checked by running tests in stress control. The solution converged in one iteration in elastic increments and two to four iterations after the yield point.

\subsection{Indentation in bone}

In order to show the abilities of the new constitutive model, a nanoindentation experiment using a Berkovich tip in cortical bone was simulated and compared to experimental results from a previous indentation study on cortical shell of human vertebral bone by Mazza (2008). The tip geometry was modeled by an equivalent conical tip with a semivertical angle of $70,3^{\circ}$. The tip of the cone was rounded with a radius of $100 \mathrm{~nm}$. This coincides with a realistic tip geometry of common indenters. Only one quarter of the bone halfspace was modeled by a sufficiently large hexahedron with the dimensions $100 \mu \mathrm{m} \times 100 \mu \mathrm{m} \times 100 \mu \mathrm{m}$ following the suggestions of Poon et al. (2008) and symmetry boundary conditions were applied in the $y-z$ and $x-z$ planes. The bottom nodes were constrained in the testing direction $(z)$. The conical indenter is modeled as an analytical rigid body of revolution. Contact was defined between the conical tip and the surface of the tested bone specimen. The rigid tip acts as master surface, the bone surface as slave. In direction normal to the surfaces, hard contact was implemented using a penalty method. No friction was defined in the tangential direction.

The halfspace was meshed using linear hexahedral elements with reduced integration and enhanced hourglass control. As large deformations and rotations were anticipated to appear in the vicinity of the nanoindentation, geometrically nonlinear analysis was activated. In order to avoid numerical problems due to element distortion, arbitrary lagrangean eulerian (ALE) remeshing was activated. In this technique, the displacement is mapped in the lagrangean, the eulerian, and the ALE domain. The displacement increments take place in the lagrangean configuration. In order to avoid excessive mesh distortion, the nodes are allowed to move with respect to the material during mesh sweeps. An advection step is then performed in the Eulerian domain to map the solution from the old to the new mesh.

The model contains approximately 9,250 elements. As the gradients of the field variables are comparatively large near the indentation and low with increasing distance to the tip, the edges of the cube were seeded with a bias toward the indentation. This allowed to have a finer mesh near the indenter tip while maintaining a relatively small number of elements. Figure 5 shows the setup of the indentation model.

The simulations were run in load control with the same parameters as the experimental setup. The load in z-direction on the conical tip was increased linearly at a rate of $60 \mathrm{mN} / \mathrm{min}$ until the holding force was reached. The linear loading was followed by a holding time of $60 \mathrm{~s}$ and

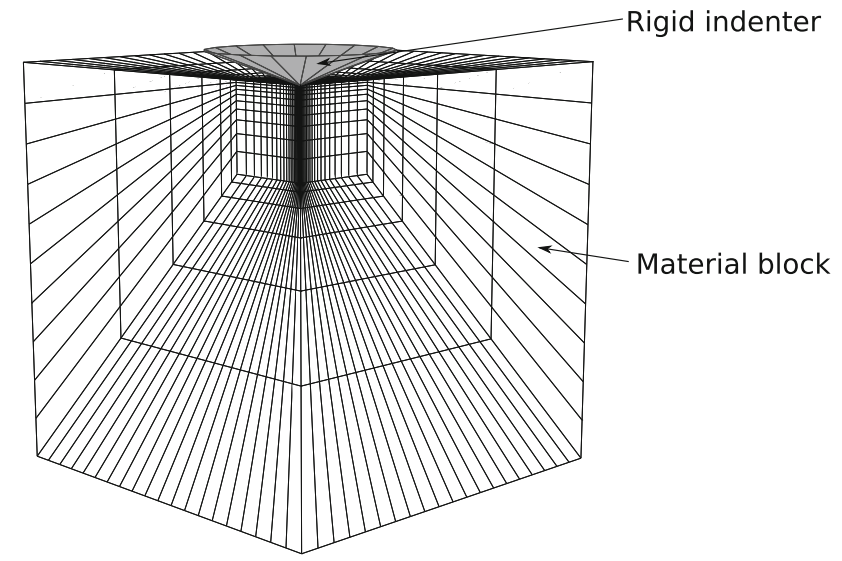

Fig. 5 Finite element indentation model

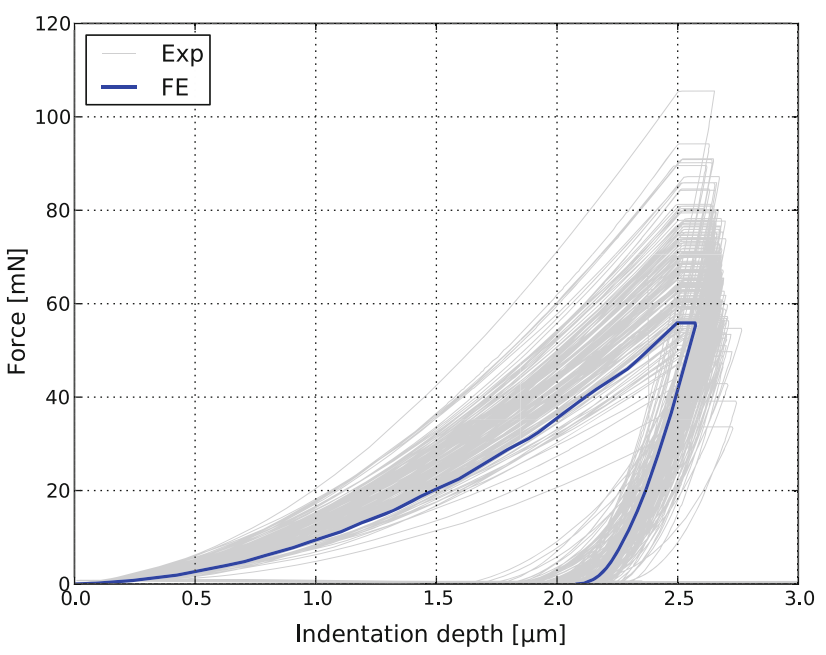

Fig. 6 Indentation curve for the tested material model

a linear unloading at the same rate. An average orthotropic stiffness tensor for cortical bone measured during the experimental indentation study (Mazza 2008) was used as stiffness input for the model. The rate-dependent yield criterion was implemented using a polynomial flow rule (see "Appendix" for details). Figure 6 compares the resulting force-depth curve for the simulation of a nanoindentation in bone using the newly proposed constitutive model with the experimental results of Mazza (2008).

The simulation was able to reach indentation depths of $2.5 \mu \mathrm{m}$ with convergence of the solution after two to six equilibrium iterations in increments where plastic deformation occurred. Large deformations were handled by the model without the occurrence of instabilities. Table 2 compares the experimentally measured indentation modulus $E_{I T}$, maximum depth $h_{\text {max }}$, residual depth $h_{\text {res }}$, elastic work $W_{\text {elast }}$, and the ratio of elastic to plastic work $W_{\text {elast }} / W_{\text {plast }}$ for cortical bone with the numerical results.

The resulting force-displacement curve is consistent with the experimental findings on bone showing inelastic 
Table 2 Comparison of experimental (Mazza 2008) with numerical results

\begin{tabular}{lllll}
\hline Measurement & Exp. Median & Exp. Min. & Exp. Max. & Model \\
\hline$E_{I T}(\mathrm{GPa})$ & 15.79 & 8.76 & 21.79 & 14.88 \\
$h_{\max }(\mu \mathrm{m})$ & 2.68 & 2.61 & 2.77 & 2.58 \\
$h_{\text {res }}(\mu \mathrm{m})$ & 1.94 & 0.85 & 2.21 & 2.08 \\
$W_{\text {elast }}$ & 13,664 & 6,100 & 23,807 & 9,652 \\
$W_{\text {elast }}(p J) / W_{\text {plast }}$ & 0.256 & 0.16 & 0.44 & 0.232 \\
\hline
\end{tabular}

deformation, creep, and reduced unloading stiffness. Indentation modulus, residual depth, elastic work, and the ratio of elastic to plastic work of the simulated indentation were within the experimental range reported by Mazza (2008). Maximum depth was slightly underestimated. No pile-up occurred around the indentation site in the model, which is also consistent with the behavior of bone seen in nanoindentation. The polynomial viscoplasticity proved to be a fast and stable constitutive model.

\section{Discussion}

An new anisotropic constitutive model for bone has been proposed that is able to capture both the elastic and the post-yield mechanical behavior of lamellar bone. It features anisotropic elasticity based on a multiscale homogenization scheme, viscoplasticity, and damage. After yielding, bone shows two mechanisms of energy dissipation: rate-dependent inelastic deformation and damage. There is evidence that these mechanisms exist already at the fibril level (Gupta et al. 2005, 2006; Hansma et al. 2005). Therefore, we believe that the proposed phenomenological model can potentially capture the post-yield behavior of bone at length scales from the fibril array to the organ level. It was hypothesized that, due to the nanogranular nature of the mineral matrix, the cohesive behavior caused by the organic phase (Tai et al. 2006) and the porosity present at all length scales (Fratzl and Weinkamer 2007), an eccentric elliptical yield criterion (Maghous et al. 2009 ) is the best choice for bone. Nonlinear isotropic hardening was implemented in order to capture the post-yield behavior. This is a reasonable choice as long as the applied loads are proportional. A micromechanics-based multiscale homogenization scheme proposed by Reisinger et al. $(2010,2011)$ has been used to obtain the elastic properties. Viscoplasticity was implemented by means of the continuous Perzyna formulation (1995) allowing for a smooth transition from rate-independent elasto-plasticity to viscoplasticity. Damage is modeled by a scalar function coupled to the plastic loading history. A polynomial flow rule was proposed in order to describe the rate-dependent post-yield behavior of lamellar bone. A numerical algorithm to perform the back projection on the rate-dependent yield surface was developed and implemented in the commercial finite element solver Abaqus/Standard as a user subroutine UMAT. A consistent tangent operator has been derived and implemented in order to assure rapid convergence.

The correct implementation of the algorithm as well as the convergence of the model were tested by means of strainand stress-based single element tests. The model showed the expected stress-strain behavior and converged up to large strains for all applied load cases. A finite element simulation of a Berkovich indentation in bone was performed in order to show the abilities of the newly developed constitutive model. Comparison to experimental results (Mazza 2008) verified that the model is able to capture the behavior of bone during indentation experiments.

Advantages of the model include: The presented formulation is very general. Due to the introduction of an isotropic yield surface in strain space, only three independent material properties are needed in addition to the elastic properties to define an anisotropic yield surface for bone, thus simplifying material identification. The combination of anisotropic elasticity with viscoplasticity and damage based on an elliptical yield surface makes the model feasible for use at multiple levels of bone hierarchy reaching from the fibril to the macroscopic level. By coupling a phenomenological postyield model to a multiscale homogenization approach predicting elastic properties, an efficient and powerful tool has been proposed to assess the mechanical behavior of bone at several length scales. The inclusion of viscoplasticity makes it possible to account for strain-rate effects as reported by Gupta et al. (2007), Gupta and Zioupos (2008) and to assess creep or relaxation behavior as seen during nanoindentation experiments.

Limitations of the model include the modeling of damage by a scalar function. As the model is supposed to work on several length scales that show different mechanisms reducing the elastic stiffness tensor most of which are not completely understood, this seemed to be a reasonable approach. Also, the model describes the post-yield behavior of bone in a continuum mechanics framework, it does not account for the exact mechanisms at the submicron scale. However, as the activated volume of the dissipative processes in bone was reported in the range from 0.64 to $1.0 \mathrm{~nm}^{3}$ by Gupta et al. (2007) and the model is supposed to reflect the mechanical behavior of bone starting at the fibril array level with representative volumes several orders of magnitude larger, this approach is justified. The assumption of an isotropic yield surface in strain space is very strong; however, given the lack of reliable data at several length scales, it is a reasonable starting point. A second limitation is the use of isotropic hardening that might not be able to catch some effects, especially during cyclic testing combining tension and compression. This limits the model's predictive capabilities 
to proportional loading, which are however the most common types of loading encountered in the body. Introduction of mixed hardening in the future would be desirable.

Both cortical and trabecular bone show inelastic deformation and a reduction in the elastic stiffness tensor after the yield point as well as pressure-dependent yield properties that differ in tension and compression (Yeni et al. 2004). The porosity in trabecular bone is considerably higher than in cortical bone; however, the underlying dissipative mechanisms remain similar. There is evidence that the yield strains for trabecular bone can be captured rather well by an eccentric isotropic elliptical yield criterion in strain space (Gross 2010). Therefore, the proposed model may be expanded to describe the homogenized response of trabecular bone in the future using fabric elasticity relationships (Zysset and Curnier 1995) and a different set of parameters for the yield surface and post-yield properties. Since a general nonlinear hardening function may be implemented in the model, hardening/softening behavior characteristic of the mechanical response of trabecular bone to compression (Charlebois et al. 2010) may be implemented in the current model.

\section{A Gradients and derivatives}

A.1 Polynomial flow rule

$$
\begin{aligned}
\dot{\lambda} & =\frac{1}{\eta}\left(Y^{2}+m Y\right) \\
\psi^{-1}(\eta \dot{\lambda}) & =-\frac{m}{2}+\left(\frac{m^{2}}{4}+\eta \dot{\lambda}\right)^{\frac{1}{2}}
\end{aligned}
$$

\section{A.2 Residual of elastic strains}

$$
\begin{aligned}
\boldsymbol{R}(\boldsymbol{S}, \Delta \kappa)= & \frac{\mathbb{E}}{1-D\left(\kappa_{0}+\Delta \kappa\right)}\left(\boldsymbol{S}-\boldsymbol{S}^{T}\right) \\
& +\frac{D\left(\kappa_{0}+\Delta \kappa\right)-D\left(\kappa_{0}\right)}{1-D\left(\kappa_{0}+\Delta \kappa\right)}\left(\boldsymbol{E}-\boldsymbol{E}_{0}^{p}\right) \\
& +\Delta \kappa \boldsymbol{N}^{p} \\
\nabla_{\boldsymbol{S}} \boldsymbol{R}= & \frac{\mathbb{E}}{1-D}+\Delta \kappa \nabla_{\boldsymbol{S}} \boldsymbol{N}^{p} \\
\frac{\partial \boldsymbol{R}}{\partial \Delta \kappa}= & \frac{D^{\prime}}{(1-D)^{2}}\left(\mathbb{E}\left(\boldsymbol{S}-\boldsymbol{S}^{T}\right)+\left(1-D_{0}\right)\left(\boldsymbol{E}-\boldsymbol{E}_{0}^{p}\right)\right) \\
& +\boldsymbol{N}^{p}+\Delta \kappa \frac{\partial \boldsymbol{N}^{p}}{\partial \Delta \kappa}
\end{aligned}
$$

A.3 Direction of plastic flow

$$
\boldsymbol{N}^{p}(\boldsymbol{S}, \Delta \kappa)=\frac{\mathbb{A}\left(\boldsymbol{S}-r\left(\kappa_{0}+\Delta \kappa\right) \boldsymbol{A}\right)}{\left\|\mathbb{A}\left(\boldsymbol{S}-r\left(\kappa_{0}+\Delta \kappa\right) \boldsymbol{A}\right)\right\|}
$$

$$
\begin{aligned}
\nabla_{\boldsymbol{S}} \boldsymbol{N}^{p} & =\frac{\mathbb{A}-\boldsymbol{N}^{p} \otimes \mathbb{A} \boldsymbol{N}^{p}}{\|\mathbb{A}(\boldsymbol{S}-\boldsymbol{A})\|} \\
\frac{\partial \boldsymbol{N}^{p}}{\partial \Delta \kappa} & =-\frac{r^{\prime} \mathbb{A} \boldsymbol{A}-\boldsymbol{N}^{p}\left(r^{\prime} \mathbb{A} \boldsymbol{A}: \boldsymbol{N}\right)}{\|\mathbb{A}(\boldsymbol{S}-\boldsymbol{r A})\|}
\end{aligned}
$$

A.4 Continuum rate-independent yield surface

$$
\begin{aligned}
Y(\boldsymbol{S}, \kappa) & =\sqrt{(\boldsymbol{S}-r(\kappa) \boldsymbol{A}): \mathbb{F}(\boldsymbol{S}-r(\kappa) \boldsymbol{A})}-r(\kappa) \\
\nabla_{S} Y & =\frac{\mathbb{A}(\boldsymbol{S}-r \boldsymbol{A})}{\sqrt{(\boldsymbol{S}-r \boldsymbol{A}): \mathbb{A}(\boldsymbol{S}-r \boldsymbol{A})}} \\
\frac{\partial Y}{\partial \kappa} & =-\frac{r^{\prime} \boldsymbol{S} \mathbb{A}-r r^{\prime} \boldsymbol{A} \mathbb{A}}{\sqrt{(\boldsymbol{S}-r \boldsymbol{A}): \mathbb{A}(\boldsymbol{S}-r \boldsymbol{A})}}-r^{\prime}
\end{aligned}
$$

A.5 Algorithmic rate-dependent yield surface

$$
\begin{aligned}
\bar{Y}(\boldsymbol{S}, \Delta \kappa)= & Y\left(\boldsymbol{S}, \kappa_{0}+\Delta \kappa\right)+\frac{m}{2}-\left(\frac{m^{2}}{4}+\frac{\eta}{\Delta t} \frac{\Delta \kappa}{h}\right)^{\frac{1}{2}} \\
\nabla_{\boldsymbol{S}} \bar{Y}= & \frac{\mathbb{A}(\boldsymbol{S}-r \boldsymbol{A})}{\sqrt{(\boldsymbol{S}-r \boldsymbol{A}): \mathbb{A}(\boldsymbol{S}-r \boldsymbol{A})}} \\
& +\frac{1}{2} \frac{\eta}{\Delta t} \frac{\Delta \kappa}{h^{2}} \nabla_{\boldsymbol{S}} h\left(\frac{m^{2}}{4}+\frac{\eta}{\Delta t} \frac{\Delta \kappa}{h}\right)^{-\frac{1}{2}} \\
\frac{\partial \bar{Y}}{\partial \Delta \kappa}= & -\frac{r^{\prime} \boldsymbol{S} \mathbb{A}-r r^{\prime} \boldsymbol{A} \mathbb{A} \boldsymbol{A}}{\sqrt{(\boldsymbol{S}-r \boldsymbol{A}): \mathbb{A}(\boldsymbol{S}-r \boldsymbol{A})}}-r^{\prime} \\
& -\frac{1}{2} \frac{\eta}{\Delta t} \frac{h-\Delta \kappa \frac{\partial h}{\partial \Delta \kappa}}{h^{2}}\left(\frac{m^{2}}{4}+\frac{\eta}{\Delta t} \frac{\Delta \kappa}{h}\right)^{-\frac{1}{2}}
\end{aligned}
$$

A.6 Norm of $\nabla_{S} Y$

$$
\begin{aligned}
h(\boldsymbol{S}, \Delta \kappa)= & \frac{\left\|\mathbb{A}\left(\boldsymbol{S}-r\left(\kappa_{0}+\Delta \kappa\right) \boldsymbol{A}\right)\right\|}{\sqrt{\left(\boldsymbol{S}-r\left(\kappa_{0}+\Delta \kappa\right) \boldsymbol{A}\right) \mathbb{A}\left(\boldsymbol{S}-r\left(\kappa_{0}+\Delta \kappa\right) \boldsymbol{A}\right)}} \\
\nabla_{S} h= & \frac{\mathbb{A} \boldsymbol{N}^{p}}{\sqrt{(\boldsymbol{S}-r \boldsymbol{A}) \mathbb{A}(\boldsymbol{S}-r \boldsymbol{A})}} \\
& -\frac{\|\mathbb{F}(\boldsymbol{S}-r \boldsymbol{A})\|(\mathbb{A}(\boldsymbol{S}-r \boldsymbol{A}))}{((\boldsymbol{S}-r \boldsymbol{A}): \mathbb{A}(\boldsymbol{S}-r \boldsymbol{A}))^{\frac{3}{2}}} \\
\frac{\partial h}{\partial \Delta \kappa}= & \frac{-r^{\prime} \mathbb{A} \boldsymbol{A}: \boldsymbol{N}^{p}}{\sqrt{(\boldsymbol{S}-r \boldsymbol{A}) \mathbb{A}(\boldsymbol{S}-r \boldsymbol{A})}} \\
& -\frac{\left.\|\mathbb{F}(\boldsymbol{S}-r \boldsymbol{A})\|\left(r^{\prime} \boldsymbol{S} \mathbb{A}-r r^{\prime} \boldsymbol{A} \mathbb{A} \boldsymbol{A}\right)\right)}{((\boldsymbol{S}-r \boldsymbol{A}): \mathbb{A}(\boldsymbol{S}-r \boldsymbol{A}))^{\frac{3}{2}}}
\end{aligned}
$$

\section{References}

Bushby AJ, Ferguson VL, Boyde A (2004) Nanoindentation of bone: comparison of specimens tested in liquid and embedded in polymethylmethacrylate. J Mater Res 19:249-259 
Carnelli D, Gastaldi D, Sassi V, Contro R, Ortiz C, Vena P (2010) A finite element model for direction-dependent mechanical response to nanoindentation of cortical bone allowing for anisotropic postyield behavior of the tissue. J Biomech Eng 132(8):081008

Carosio A, Willam K, Etse G (2000) On the consistency of viscoplastic formulations. Int J Solids Struct 37(48-50):7349-7369

Chaboche JL (2008) A review of some plasticity and viscoplasticity constitutive theories. Int J Plast 24:1642-1693

Charlebois M, Jirasek M, Zysset P (2010) A nonlocal constitutive model for trabecular bone softening in compression. Biomech Model Mechanobiol 9:597-611

Cowin SC (1979) On the strength anisotropy of bone and wood. J Appl Mech 46(4):832-838

Cowin S, Mehrabadi M (1995) Anisotropic symmetries of linear elasticity. Appl Mech Rev 48:247-285

Etse G, Carosio A (1999) Constitutive equations and numerical approaches in rate dependent material formulations, MECOM

Fondrk MT, Bahniuk EH, Davy DT (1999) A damage model for nonlinear tensile behavior of cortical bone. J Biomech Eng 121:533-541

Fratzl P, Weinkamer R (2007) Nature's hierarchical materials. Prog Mater Sci 52(8):1263-1334

Garcia D (2006) Elastic plastic damage laws for cortical bone. Ph.D. thesis, Ecole Polytechnique Federale de Lausanne

Garcia D, Zysset P, Charlebois M, Curnier A (2009) A three-dimensional elastic plastic damage constitutive law for bone tissue. Biomech Model Mechanobiol 8(2):149-165

Green AE, Naghdi PM (1965) A general theory of an elastic-plastic continuum. Arch Ration Mech Anal 18(4):251-281

Gross T (2010) The effects of heterogeneous mineralization on the elastic and yield properties of human cancellous bone. Diploma Thesis, Vienna University of Technology

Gupta H, Zioupos P (2008) Fracture of bone tissue: the 'hows' and the 'whys'. Med Eng Phys 30(10):1209-1226

Gupta HS, Wagermaier W, Zickler GA, Raz-Ben Aroush D, Funari SS, Roschger P, Wagner HD, Fratzl P (2005) Nanoscale deformation mechanisms in bone. Nano Lett 5(10):2108-2111

Gupta H, Wagermaier W, Zickler G, Hartmann J, Funari S, Roschger P, Wagner H, Fratzl P (2006) Fibrillar level fracture in bone beyond the yield point. Int J Fract 139:425-436

Gupta HS, Fratzl P, Kerschnitzki M, Benecke G, Wagermaier W, Kirchner HO (2007) Evidence for an elementary process in bone plasticity with an activation enthalpy of $1 \mathrm{eV}$. J R Soc Interface 4(13):277-282

Hansma P, Fantner G, Kindt J, Thurner P, Schitter G, Turner P, Udwin $S$, Finch M (2005) Sacrificial bonds in the interfibrillar matrix of bone. J Musculoskelet Neuronal Interact 5(4):313-315

Hellmich C, Ulm FJ (2002) Are mineralized tissues open crystal foams reinforced by crosslinked collagen?-some energy arguments. J Biomech 35(9):1199-1212

Hengsberger S, Kulik A, Zysset P (2002) Nanoindentation discriminates the elastic properties of individual human bone lamellae under dry and physiological conditions. Bone 30(1):178-184

Keyak JH, Rossi SA (2000) Prediction of femoral fracture load using finite element models: an examination of stress- and strain-based failure theories. J Biomech 33(2):209-214

Kuhn HW, Tucker AW (1951) Nonlinear programming. In: Proceedings of 2nd Berkeley symposium. University of California Press, pp 481-492

Lucchini R, Carnelli D, Ponzoni M, Bertarelli E, Gastaldi D, Vena P (2011) Role of damage mechanics in nanoindentation of lamellar bone at multiple sizes: experiments and numerical modeling. J Mech Behav Biomed Mater 4(8):1852-1863

Maghous S, Dormieux L, Barthèlèmy J (2009) Micromechanical approach to the strength properties of frictional geomaterials. Eur J Mech A Solids 28(1):179-188
Mazza G (2008) Anisotropic elastic properties of vertebral bone measured by microindentation. Diploma thesis, Politecnico di Milano

Natali A, Carniel E, Pavan P (2008) Constitutive modelling of inelastic behaviour of cortical bone. Med Eng Phys 30(7):905-912

Nemat-Nassar S, Mori M (1993) Micromechanics: overall properties of heterogeneous materials. Elsevier Science Publishers, The Netherlands

Perzyna P. (1966) Fundamental problems in viscoplasticity. Elsevier, The Netherlands 243-377

Ponthot JP (1995) Radial return extensions for visco-plasticity and lubricated friction. In: SMIRT-13 international conference on structural mechanics and reactor technology

Poon B, Rittel D, Ravichandran G (2008) An analysis of nanoindentation in linearly elastic solids. Int J Solids Struct 45(24):60186033

Rakatomanana RL, Curnier A, Leyvraz PF (1991) An objective elastic plastic model and algorithm applicable to bone mechanics. Eur J Mech A Solids 10(3):327-342

Reisinger A, Pahr D, Zysset P (2010) Sensitivity analysis and parametric study of elastic properties of an unidirectional mineralized bone fibril-array using mean field methods. Biomech Model Mechanobiol 9:499-510

Reisinger A, Pahr D, Zysset P (2011) Elastic anisotropy of bone lamellae as a function of fibril orientation pattern. Biomech Model Mechanobiol 10:67-77

Shih CF, Lee D (1978) Further developments in anisotropic plasticity. J Eng Mater Technol 100(3):294-302

Simo JC, Ju JW (1987) Strain- and stress-based continuum damage models-I. Formulation. Int J Solids Struct 23(7):821-840

Spiesz EM (2011) Experimental and computational micromechanics of mineralized tendon and bone. Ph.D. thesis, Vienna University of Technology

Spiesz EM, Reisinger AG, Roschger P, Zysset PK (2011) Experimental validation of a multiscale model of mineralized collagen fibers at two levels of hierarchy. Osteoporos Int 22:561-666

Sugawara Y, Kamioka H, Honjo T, Tezuka K, Takano-Yamamoto T (2005) Three-dimensional reconstruction of chick calvarial osteocytes and their cell processes using confocal microscopy. Bone 36(5):877-883

Tai K, Ulm FJ, Ortiz C (2006) Nanogranular origins of the strength of bone. Nano Lett 6(11):2520-2525

Voyiadjis G, Peters R (2010) Size effects in nanoindentation: an experimental and analytical study. Acta Mech 211:131-153

Weiner S, Arad T, Sabanay I, Traub W (1997) Rotated plywood structure of primary lamellar bone in the rat: orientations of the collagen fibril arrays. Bone 20(6):509-514

Weiner S, Wagner HD (1998) The material bone: structure-mechanical function relations. Annu Rev Mater Sci 28(1):271-298

Weiner S, Traub W, Wagner H (1999) Lamellar bone: structure-function relations. J Struct Biol 126(3):241-255

Wolfram U, Wilke HJ, Zysset PK (2010) Rehydration of vertebral trabecular bone: influences on its anisotropy, its stiffness and the indentation work with a view to age, gender and vertebral level. Bone 46(2):348-354

Yeni Y, Dong X, Fyhrie D, Les C (2004) The dependence between the strength and stiffness of cancellous and cortical bone tissue for tension and compression: extension of a unifying principle. Bio-Med Mater Eng 14(3):303-310

Zhang J, Niebur GL, Ovaert TC (2008) Mechanical property determination of bone through nano- and micro-indentation testing and finite element simulation. J Biomech 41(2):267-275

Zhang J, Michalenko MM, Kuhl E, Ovaert TC (2010) Characterization of indentation response and stiffness reduction of bone using a continuum damage model. J Mech Behav Biomed Mater 3(2):189202 
Zinkiewicz OC, Valliapan S, King IP (1969) Elastoplastic solutions of engineering problems initial stress, finite element approach. Int $\mathbf{J}$ Num Methods Eng 1:75-100

Zysset PK (1994) A constitutive law for trabecular bone. Ph.D. thesis, Ecole Polytechnique Federale de Lausanne

Zysset PK, Curnier A (1995) An alternative model for anisotropic elasticity based on fabric tensors. Mech Mater 21(4):243-250
Zysset PK, Guo EX, Hoffler EC, Moore KE, Goldstein SA (1999) Elastic modulus and hardness of cortical and trabecular bone lamellae measured by nanoindentation in the human femur. J Biomech 32(10):1005-1012 